\title{
Amniotic Fluid Selenium Status in Pre-Eclampsia
}

\begin{tabular}{|c|c|}
\hline A.C. & Roy $^{\mathrm{a}}$ \\
\hline S.S. & Ratnam $^{\mathrm{a}}$ \\
\hline R. & Karunanithy \\
\hline
\end{tabular}

Departments of ${ }^{\mathrm{a} O b s t e t r i c s}$ and Gynaecology, and ${ }^{\mathrm{b}}$ Pharmacy, National University of Singapore, Republic of Singapore

\section{Key Words}

Selenium status

Amniotic fluid

Pre-eclampsia

Glutathione peroxidase

Lipid peroxides

\section{Abstract}

Selenium levels were measured in amniotic fluid samples obtained from 60 normal pregnant and 40 pre-eclamptic women closely matched for age and gestation using the hydride generation technique coupled with atomic absorption spectroscopy. The mean \pm SD levels in the normal and pre-eclamptic groups were $6.8 \pm 3.7$ and $6.7 \pm 2.2 \mathrm{ng} / \mathrm{ml}$, respectively. The difference in the values of amniotic fluid selenium between these two groups of patients was not statistically significant $(p>0.5)$.

Dr. A.C. Roy, Department of Obstetrics and Gynaecology, National University of Singapore, National University Hospital, Lower Kent Ridge Road, Singapore 0511 (Republic of Singapore)

Materials and Methods

Amniotic fluid aliquots were obtained with consent from 60 normal pregnant women (median age, 28 years) and 40 pre-eclamptic women (median age, 30 years) between 34 and 42 weeks of gestation. The mean \pm SD gestational age for normal and pre-eclamptic groups was $37.2 \pm 1.5$ and $36.3 \pm 1.8$ respectively ( $\mathrm{p}>0.5$ ). Diagnosis of pre-eclampsia was based on the blood pressure constantly over 140/90 mm Hg (range, 130/90-170/110 mm Hg) with a mild proteinuria and/or oedema; none of them had a history of hypertension or kidney disease before the pregnancy. Patients with other complications such as diabetes, multiple or abnormal fetuses, chronic infections, metabolic, endocrine, nutritional or haematolog-ical disorders were excluded from the study. None of the subjects in both groups were receiving any medication other than routine prenatal vitamins and iron tablets. Gestation at the time of sample collection was determined from the last menstrual period and uterine size.

About $1-2$ ml of amniotic fluid, obtained carefully by transab-dominal amniocentesis or hind water amniotomy at induction after ultrasonographic localization of the fetus/placenta, was immediately centrifuged at $1,500 \mathrm{~g}$ and $4^{\circ} \mathrm{C}$ for lOmin, and the supernatant stored at $-20^{\circ} \mathrm{C}$ until analyzed. Samples containing erythrocytes or meconium were excluded from the study. The Se concentration was determined by hydride generation technique coupled with atomic absorption spectrophotometer equipped with a Se hollow cathode lamp and a Perkin-Elmer MHS 10 vapour generation accessory as previously described [2]. The Introduction 
Selenium deficiency has been described as a characteristic feature of a variety of illnesses such as cardiovascular disease, cancer, kwashiorkor and keshan disease, and sudden infant death syndrome [1, 2]. The essential trace element is also required for normal intrauterine growth and development of the fetus. In animals, during pregnancy and lactation, Se deficiency results in a wide spectrum of physiological and pathological conditions including retarded growth, resorption and even death of the fetus, retained placenta, and conditions such as white muscle disease in the neonates [1, 2].

The literature on the effects of Se deficiency in pregnant women is, however, scanty. Plasma level of Se has been found to be significantly lower in pregnant than in nonpregnant women [3, 4], and recent studies have shown that vascular (whole blood, plasma and serum) [5, 6] and amniotic [2] fluid levels of the essential trace element decrease with the progress of pregnancy. In the present study, we measured amniotic fluid Se levels in pre-eclamptic women, and compared the results with normal pregnant women closely matched for age and gestation. 162

Roy/Ratnam/Karunanithy

Table 1. Amniotic fluid concentrations of selenium in normal pregnant and pre-eclamptic women (mean $\pm \mathrm{SD}$ )

Group $\mathrm{n} \quad$ Weeks of gestation Se level, $\mathrm{ng} / \mathrm{ml}$

Normal $\quad 60 \quad 37.2 \pm 1.5(34-42) \quad 6.8 \pm 3.7$

Pre-eclamptic $40 \quad 36.3 \pm 1.8(34-40) \quad 6.7 \pm 2.2$

$\mathrm{p}>0.5$. Ranges in parentheses.

intra- and interassay coefficients of variation were 1.8 and 3.5\%, respectively. The statistical significance was evaluated by Student's t test (two-tailed) with a p value $<0.05$ considered significant.

Results

The levels of Se in the amniotic fluid samples obtained from normal pregnant and pre-eclamptic women between 34 and 42 weeks of gestation are shown in table 1 . There was no significant difference $(p>0.5)$ in the levels of amniotic fluid Se between normal and toxaemic pregnancy. Discussion

Selenium is a normal constituent of glutathione per-oxidase, an enzyme responsible for the catalytic detoxification of hydrogen peroxide and lipid peroxides via reduced glutathione. The essential trace element is located at the active site and participates in the catalytic process of the enzyme, preventing peroxidative tissue damage. Most of the Se-deficient conditions in animals are characterized by the free-radical-induced peroxida-tion of membrane-bound polyunsaturated fatty acids $[7,8]$.

Recently, plasma levels of various free-radical oxidation products have been shown to increase progressively throughout pregnancy, and decline immediately after parturition [9]. A direct correlation between the free-radicals and blood pressure in some cases of pre-eclamp-sia has also been established [9]. Therefore, there is a possibility that lipid peroxides may be involved in the pathogenesis of toxaemic pregnancy. Although this study was unable to show any change in the amniotic fluid Se level in pre-eclampsia, a role for Se at the cellular level as a preventive factor for toxaemia should not be discounted.

Acknowledgements

We are grateful to Dr RNV Prasad and Dr OAC Viegas for providing amniotic fluid samples, and wish to thank Miss Ng Sek Eng for technical assistance. 


\section{References}

Shamberger, R.J.: Selenium; in Frieden, Biochemistry of the essential ultratrace elements, pp. 201-237 (Plenum Press, New York 1984).

Karunanithy, R.; Roy, A.C.; Ratnam, S.S.: Selenium status in pregnancy: studies in amniotic fluid from normal pregnant women. Gynecol. obstet. Invest. 27: 148-150 (1989).

Rudolph, N; Wong, S.L.: Selenium and glutathione peroxidase activity in maternal and cord plasma and red cells. Pediat. Res. 12: 789-792 (1978).

Behne, D.; Wolters, W:: Selenium content and glutathione peroxidase activity in the plasma and erythrocytes of non-pregnant and pregnant women. J. clin. Chem. clin. Biochem. 17: 133-135 (1979).

Butler, J.A.; Whanger, P.D.; Tripp, MJ.: Blood selenium and glutathione peroxidase activity in pregnant women: comparative assays in primates and other animals. Am. J. clin. Nutr. 36: 15-23 (1982).

Hyvonen-Dabek, M.; Nikkinen-Vilkki, P.; Dabek, J.T.: Selenium and other elements in human maternal and umbilical serum, as determined simultaneously by protein-induced x-ray emission. Clin. Chem. 30: 529-533 (1984).

Tappel, A.L.: Lipid peroxidation damage to cell components. Fed. Proc. 32: 1870-1874 (1973). Jamall, I.S.; Haldar, D.; Wadewitz, A.G.: Effects of dietary selenium on lipid peroxidation, mitochondrial function and protein profiles in the heart of the myopathic Syrian golden hamster (BIO 14.6). Biochem. biophys. Res. Commun. 144: 815-820 (1987).

Wickens, D.; Wilkins, M.H.; Lunec, J.; Ball, G.; Dormandy, T.L.: Free-radical oxidation (peroxidation) products in plasma in normal and abnormal pregnancy. Ann. clin. Biochem. 18: 158-162(1981). 